\title{
Cinética de secagem de acerola em leito de espuma e ajuste de modelos matemáticos
}

\author{
Foam-mat drying kinetics for acerola and adjustment of the mathematical models
}

\section{Cintia da Silva Araújo ${ }^{1 *}$, Leandro Levate Macedo', Wallaf Costa Vimercati ${ }^{1}$, Sérgio Henriques Saraiva ${ }^{1}$, Anderson do Nascimento Oliveira', Luciano José Quintão Teixeira'}

${ }_{1}^{1}$ Universidade Federal do Espírito Santo (UFES), Departamento de Engenharia de Alimentos, Alegre/ES, Brasil

\section{${ }^{*}$ Corresponding Author}

Cintia da Silva Araújo, Universidade Federal do Espírito Santo (UFES), Centro de Ciências Agrárias e Engenharias, Departamento de Engenharia de Alimentos, Alto Universitário, s/n, CEP: 29500-000, Alegre/ES - Brasil, e-mail: araujo.s.cintia@gmail.com

Cite as: Foam-mat drying kinetics for acerola and adjustment of the mathematical models. Braz. J. Food Technol., v. 20, e2016152, 2017.

Received: Oct. 24, 2016; Accepted: June 28, 2017

\section{Resumo}

A acerola (Malpighia emarginata DC) é uma fruta rica em nutrientes, principalmente vitamina C. No entanto, a perecibilidade do fruto in natura é alta. Desta forma, o processamento do fruto torna-se indispensável. Diante disto, o presente trabalho teve o objetivo de estudar a etapa de secagem da polpa de acerola em leito de espuma, além de avaliar o efeito da temperatura sobre a cinética de secagem da polpa de acerola e ajustar modelos matemáticos para descrever o processo. O experimento foi conduzido em três repetições. A espuma foi elaborada com $250 \mathrm{~mL}$ da polpa de acerola adicionada de $4 \%$ do agente espumante, sendo, então, espalhada uniformemente em bandejas de inox. As temperaturas do ar de secagem selecionadas foram de $50^{\circ} \mathrm{C}, 55^{\circ} \mathrm{C}, 60^{\circ} \mathrm{C}, 65^{\circ} \mathrm{C}$ e $70^{\circ} \mathrm{C}$. Posteriormente, as razões de umidade foram calculadas e os modelos matemáticos de Page, Henderson e Pabis e Lewis foram ajustados para cada temperatura. O modelo de Page apresentou o maior valor de coeficiente de determinação ajustado $(>0,99)$ e o menor valor do erro padrão da regressão, em todas as temperaturas $(<0,03)$. O modelo generalizado proposto apresentou coeficiente de determinação de 0,9933. Os modelos de Page e o generalizado foram adequados para descrever a etapa de secagem da polpa de acerola. Os valores de umidade dos produtos desidratados apresentaram-se em conformidade com o estabelecido pela legislação brasileira.

Palavras-chave: Malpighia emarginata DC.; Polpa de acerola; Modelo de Page; Modelo de Henderson e Pabis; Modelo de Lewis.

\section{Abstract}

Acerola (Malpighia emarginata DC) is a fruit rich in nutrients, especially vitamin C, but the in natura fruit is highly perishable and hence processing of the acerola pulp becomes indispensable. The aim of this paper was to study the foam mat drying process of acerola pulp to evaluate the effect of temperature on the drying kinetics of the pulp and fit mathematical models in order to describe the operation. The experiment was carried out with three repetitions. The foam was prepared with $250 \mathrm{~mL}$ of the pulp plus $4 \%$ of foaming agent and spread evenly in stainless steel trays. The selected drying air temperatures were $50{ }^{\circ} \mathrm{C}, 55^{\circ} \mathrm{C}, 60^{\circ} \mathrm{C}, 65^{\circ} \mathrm{C}$ and $70^{\circ} \mathrm{C}$. The moisture ratios were then calculated and the Page, Henderson and Pabis and Lewis mathematical models fitted for each temperature. The Page model showed the highest determination coefficient (>0.99) and lowest standard error for the regression at all temperatures $(<0.03)$. The proposed generalized model presented a determination coefficient of 0.9933 . The Page model and the generalized model were adequate to describe the drying process of the acerola pulp. The moisture contents of the dehydrated products were in accordance with the values established by Brazilian law.

Keywords: Malpighia emarginata DC.; Acerola pulp; Page model; Henderson and Pabis model; Lewis Model. 


\section{Introdução}

A acerola (Malpighia emarginata DC) é uma fruta tropical originária dos países da América. O consumo do fruto é fomentado pela riqueza em nutrientes, principalmente pelo alto teor de ácido ascórbico (vitamina C) e outros nutrientes, tais como niacina, riboflavina e tiamina (ASSIS et al., 2001; MEZADRI et al., 2008).

O período de vida útil da acerola é curto após a colheita, devido às reações bioquímicas de deterioração responsáveis pela perda de qualidade do fruto. Desta maneira, o processamento colabora com o aumento da vida útil, além de facilitar o transporte e agregar valor ao produto (ALVES et al., 2010).

A secagem é um processo tradicional na conservação de alimentos, responsável por diminuir a disponibilidade de água para reações de deterioração, aumentar a estabilidade do alimento e reduzir o volume e a massa do produto (RAJKUMAR et al., 2007; CELMA et al., 2011; CASARIN et al., 2016).

A secagem no leito em espuma é uma operação de desidratação pela qual alimentos líquidos ou semilíquidos são transformados em uma espuma estável, pela adição de agentes espumantes e aeração (KARIM; WAI, 1999; FELLOWS, 2006). Este método destaca-se pela fácil reconstituição em água da polpa transformada em pó, alta relação massa/volume, possibilidade de formulação com outros produtos e redução de custos com espaço de armazenamento (VIEIRA et al., 2007; MOREIRA et al., 2013).

A secagem pode ser descrita por modelos matemáticos, que possibilitam a obtenção de estimativas do tempo necessário para redução do teor de água do produto, em diferentes condições de secagem, de modo que se tornam ferramentas úteis para as tomadas de decisão e contribuem para a melhoria da eficiência do processo (KADAM; BALASUBRAMANIAN, 2011; MEZIANE, 2011; SOUSA et al., 2011). Os modelos matemáticos de Henderson e Pabis (1962), Lewis (1921) e Page (1949) são largamente adotados em processos de secagem (MEZIANE, 2011; KALETA et al., 2013).

A cinética de secagem e o ajuste de modelos matemáticos já foram estudados para diversas frutas, tais como carambola (KARIM; WAI, 1999), graviola (BAPTESTINI et al., 2015), nabo forrageiro (SOUSA et al., 2011), maçã (KALETA et al., 2013), mamão (KANDASAMY et al., 2012) e tomate (KADAM; BALASUBRAMANIAN, 2011), porém poucos estudos exploraram a acerola. Desta forma, este trabalho teve os objetivos de estudar a etapa de secagem da polpa de acerola em leito de espuma, avaliar o efeito da temperatura sobre a cinética de secagem da polpa de acerola, ajustar modelos matemáticos para descrever a operação e avaliar o efeito da temperatura sobre a umidade do produto desidratado.

\section{Material e métodos}

\subsection{Materiais}

Os frutos de acerola (Malpighia emarginata DC) foram obtidos no comércio local do município de Alegre, Espírito Santo, Brasil. O experimento foi conduzido no Laboratório do Centro de Ciências Agrárias da Universidade Federal do Espírito Santo, onde os frutos passaram por seleção, para retirada de frutos imaturos e injuriados, e foram submetidos a lavagem com água corrente e sanitização com água clorada a 200 ppm. Os frutos foram triturados em liquidificador doméstico no momento da realização das análises e o extrato foi filtrado para obtenção da polpa.

\subsection{Fluxograma metodológico}

A Figura 1 apresenta o fluxograma metodológico, fracionado em quatro etapas.

\subsection{Teste preliminar}

O teste preliminar teve o objetivo de determinar o agente espumante e a sua mínima concentração que proporcionasse a formação de uma espuma estável. Para isso, os aditivos Albumina e Emustab ${ }^{\circledR}$ foram adicionados em $250 \mathrm{~mL}$ de polpa de acerola, nas concentrações de $2 \%, 4 \%, 6 \%, 8 \%$ e $10 \%(\mathrm{~m} / \mathrm{v})$. A mistura foi agitada em uma batedeira (ARNO planetária ${ }^{\circledR}$, modelo BPAI, $280 \mathrm{~W}, 50-60 \mathrm{~Hz}$ ) por 20 minutos, para a formação da espuma. Durante os cinco minutos iniciais, foi utilizada a velocidade mínima da batedeira, e a velocidade máxima, nos 15 minutos restantes. A espuma formada foi avaliada quanto a densidade e percentual de volume drenado. A densidade da espuma foi obtida com o auxílio de uma proveta de $2.000 \mathrm{~mL}$, em que a densidade foi calculada pela razão entre o volume ocupado pela espuma e a massa da espuma. O percentual de volume drenado foi calculado pela razão entre o volume drenado e o volume total da espuma formada. A quantidade de líquido drenado foi medida utilizando-se régua milimetrada no tempo zero e no intervalo de cinco em cinco minutos, até que se completassem 30 minutos, à temperatura ambiente.

\subsection{Experimento final}

Com base no teste preliminar, o aditivo e a concentração foram determinados para a condução do experimento de secagem. Na etapa de formação da espuma, foram utilizados $250 \mathrm{~mL}$ da polpa de acerola adicionada de $4 \%$ (m/v) do agente emulsificante Emustab ${ }^{\circledR}$. O procedimento realizado para a elaboração da espuma foi o mesmo utilizado no teste preliminar.

O experimento foi realizado em três repetições. As espumas foram espalhadas uniformemente em bandejas de inox com dimensões $50 \times 40 \mathrm{~cm}$ e $1 \mathrm{~cm}$ de altura, sendo então colocadas em um secador de cabine com 


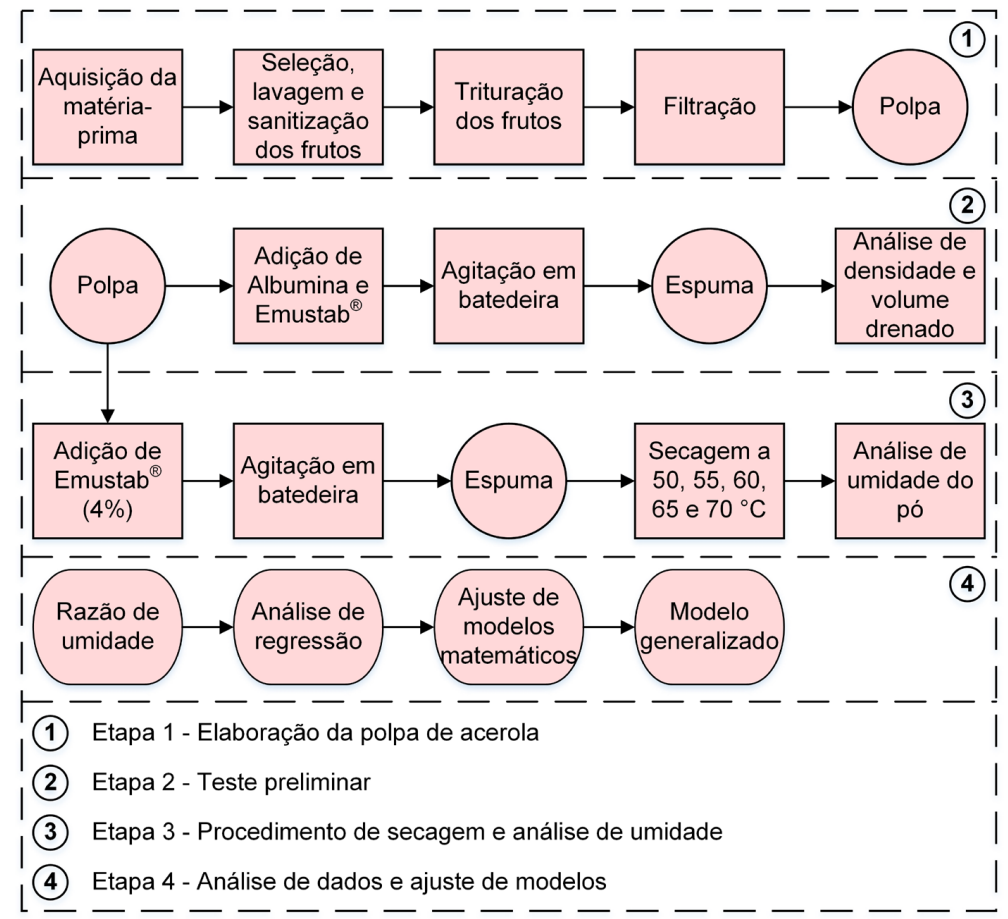

Figura 1. Fluxograma geral da condução do experimento.

circulação forçada de $\operatorname{ar}(1,5 \mathrm{~m} / \mathrm{s})$. As temperaturas do ar de secagem selecionadas foram de $50{ }^{\circ} \mathrm{C}, 55^{\circ} \mathrm{C}, 60^{\circ} \mathrm{C}$, $65^{\circ} \mathrm{C}$ e $70^{\circ} \mathrm{C}$, totalizando cinco níveis de temperatura e perfazendo, portanto, um número adequado para o estudo de um fator quantitativo. Esses valores de temperatura selecionados proporcionam menores alterações de características nutricionais e sensoriais da polpa, sendo este um intervalo comumente utilizado no método de leito em espuma (MEZIANE, 2011; BAPTESTINI et al., 2015). A massa das amostras foi registrada utilizando-se uma balança com resolução de 0,001 g, em intervalos de 15 minutos durante a primeira hora, 30 minutos durante a segunda e terceira hora, e 60 minutos até que a massa permanecesse constante.

Os cálculos para determinação da razão de umidade $(R U)$ experimental foram realizados para cada temperatura, conforme a Equação 1. A RU é definida como sendo a diferença entre a umidade, em base seca, em um dado tempo $t$ de secagem, e a umidade de equilíbrio, dividida pela diferença entre a umidade no início da secagem e a umidade de equilíbrio. Essa razão também pode ser expressa em função das massas do material sendo desidratado.

$R U=\frac{X_{t}-X_{e}}{X_{0}-X_{e}}=\frac{M_{t}-M_{e}}{M_{0}-M_{e}}$

em que $\mathbf{X}_{0}, \mathbf{X}_{\mathrm{t}}$ e $\mathbf{X}_{\mathrm{e}}$ são os valores da umidade, em base seca, da espuma antes de ser colocada no secador, no tempo thoras após o início da secagem e no equilíbrio.
$M_{0}, M_{t}$ e $M_{e}$ são as massas, em gramas, da espuma na bandeja antes de ser colocada no secador, no tempo thoras após o início da secagem e no equilíbrio, respectivamente.

Os dados experimentais de razão de umidade ao longo do tempo de secagem foram submetidos à análise de regressão pelo programa SigmaPlot 11.0, e os modelos de Page (Equação 2), Henderson e Pabis (Equação 3) e Lewis (Equação 4) foram ajustados para cada temperatura.

$$
\begin{aligned}
& R U=e^{-k t^{n}} \\
& R U=a e^{-k t} \\
& R U=e^{-k t}
\end{aligned}
$$

em que $a, k$ e $n$ são parâmetros dos modelos, e $t$ é o tempo de secagem, em horas.

Os ajustes dos parâmetros dos modelos foram feitos por meio da minimização da soma de quadrados dos desvios, usando regressão não linear.

Deste modo, o modelo que melhor descreveu a cinética de secagem foi determinado e foi avaliado o efeito da temperatura do ar de secagem sobre seus parâmetros. Um modelo matemático foi proposto para descrever esse efeito. Após a escolha das funções que melhor representassem os parâmetros, um modelo generalizado, no qual os parâmetros originais foram substituídos por essa função na cinética de secagem, foi ajustado por regressão não linear. 
Cinética de secagem de acerola em leito de espuma e ajuste de modelos matemáticos

Araújo, C. S. et al.

\subsection{Análise de umidade}

A determinação de umidade, em base úmida, dos pós obtidos nas diferentes temperaturas do ar de secagem, foi realizada por meio do analisador de umidade por infravermelho - IV 2500, marca GEHAKA, com resolução de $0,1 \%$. Para tanto, foram utilizados cinco gramas dos produtos secos.

\section{Resultados e discussão}

\subsection{Teste preliminar}

As densidades das espumas obtidas e os percentuais de volume drenado finais, ou seja, após 30 minutos, nas concentrações 2\%, 4\%, 6\%, 8\% e 10\% de Albumina e de Emustab $^{\circledR}$, são apresentados na Tabela 1.

O agente espumante Albumina não proporcionou estabilidade à espuma formada, visto que esta apresentou alto valor percentual de volume drenado, nas cinco concentrações propostas. Em contrapartida, o Emustab ${ }^{\circledR}$, em concentrações acima de $4 \%$, possibilitou a estabilidade da espuma, já que não foi observada coalescência, isto é, obteve-se zero de volume drenado. Além disso, a densidade da espuma apresentou-se suficientemente baixa, com valores inferiores aos apresentados pela mistura com Albumina. Portanto, a concentração de 4\% de Emustab ${ }^{\circledR}$ foi a escolhida para o processo de secagem.

Soares (2009), estudando a secagem em leito de espuma de araçá-boi, testou quatro diferentes aditivos, nas concentrações de 5\%, 10\%, 15\% e 20\% em massa, para formação da espuma, sendo a albumina escolhida como o melhor aditivo para a etapa de secagem, conduzida à temperatura de $70^{\circ} \mathrm{C}$. Pinto (2009) utilizou os aditivos albumina, Emustab ${ }^{\circledR}$, maltodextrina e Superliga ${ }^{\circledR}$ na caracterização da espuma de jenipapo, para secagem em leito de espuma, e concluiu que o Emustab ${ }^{\circledR}$ foi o aditivo que, nas concentrações testadas, apresentou-se como o melhor para uma posterior secagem. Dantas et al. (2008) usaram, como aditivo, o Emustab ${ }^{\circledR}$ na concentração de 7,5\%, para a obtenção de polpa de jaca em pó pela operação de secagem em leito de espuma, sendo o processo realizado a temperaturas de $60^{\circ} \mathrm{C}, 70^{\circ} \mathrm{C}, 80^{\circ} \mathrm{C}$ e $90^{\circ} \mathrm{C}$.

\subsection{Experimento final}

Os modelos matemáticos, dados pelas Equações 2, 3 e 4, foram ajustados após os cálculos para determinação das razões de umidade, ao longo do tempo de secagem. $\mathrm{Na}$ Tabela 2, são apresentados os ajustes dos modelos por meio de regressão não linear aos dados experimentais da secagem da polpa de acerola em leito de espuma, nas diferentes temperaturas do ar de secagem. O melhor modelo para a secagem apresenta valor do coeficiente de determinação ajustado $(R)$ e valor do erro padrão da regressão (S) o mais próximo de um e zero, respectivamente.

Todos os modelos apresentaram altos valores de $R$ e baixos valores de $S$. O modelo de Lewis, na temperatura de $70{ }^{\circ} \mathrm{C}$, foi o que obteve os menores valores de $R$ e os maiores valores de $S, 0,9622$ e 0,0758, respectivamente. Este modelo, dentre os testados, é o menos apropriado para descrever a etapa de secagem da polpa de acerola.

O modelo que apresentou o maior valor do coeficiente de determinação ajustado e o menor valor do erro padrão da regressão (Tabela 2), em todas as temperaturas avaliadas, foi o de Page, cujos valores de $R$ foram maiores que 0,99 para todas as temperaturas, indicando que o modelo é adequado para representar a operação (MADAMBA et al., 1996). O modelo de Page apresentou-se o mais adequado também para outros alimentos, tais como abacaxi (HOFSKY et al., 2009), maçã (KALETA et al., 2013) e tomate (CELMA et al., 2011).

O fato de o modelo de Page ter sido o mais adequado diante dos demais modelos para representar a cinética de secagem em leito de espuma da polpa de acerola também pode ser verificado na Figura 2, na qual estão representadas as curvas de secagem em diferentes temperaturas. A curva ajustada pelo modelo de Page foi a que mais se aproximou dos dados experimentais para todas as temperaturas avaliadas.

Tabela 1. Características dos agentes espumantes nas diferentes concentrações.

\begin{tabular}{cccc} 
Agente espumante & Concentração (\%) & $\begin{array}{c}\text { Pensidade da espuma (g/mL) } \\
\text { Dentual de volume } \\
\text { drenado (\%) }\end{array}$ \\
Albumina & 2 & 0,748 & 59,01 \\
& 4 & 0,632 & 23,79 \\
& 6 & 0569 & 15,18 \\
& 8 & 0,542 & 12,82 \\
Emustab $^{\circledR}$ & 10 & 0,537 & 11,25 \\
& 2 & 0,327 & 6,95 \\
& 4 & 0,214 & 0,00 \\
& 6 & 0,201 & 0,00 \\
\hline
\end{tabular}


Cinética de secagem de acerola em leito de espuma e ajuste de modelos matemáticos

Araújo, C. S. et al.

Tabela 2. Parâmetros estimados, coeficiente de determinação ajustado $(R)$ e erro padrão da regressão $(S)$, para os modelos de Page, Lewis e de Henderson e Pabis (H\&P), em diferentes temperaturas do ar de secagem.

\begin{tabular}{|c|c|c|c|c|c|}
\hline Temperatura & Modelos & $k$ & a ou $n$ & $\boldsymbol{R}^{2}{ }_{a d j}$ & $s$ \\
\hline \multirow{3}{*}{$50^{\circ} \mathrm{C}$} & Lewis & 0,3766 & - & 0,9727 & 0,0636 \\
\hline & Page & 0,2512 & 1,4319 & 0,9977 & 0,0183 \\
\hline & $H \& P$ & 0,4121 & 1,0779 & 0,9793 & 0,0550 \\
\hline \multirow{3}{*}{$55^{\circ} \mathrm{C}$} & Lewis & 0,5605 & - & 0,9750 & 0,0603 \\
\hline & Page & 0,4426 & 1,4221 & 0,9963 & 0,0231 \\
\hline & H\&P & 0,6072 & 1,0716 & 0,9792 & 0,0550 \\
\hline \multirow{3}{*}{$60^{\circ} \mathrm{C}$} & Lewis & 0,5744 & - & 0,9683 & 0,0689 \\
\hline & Page & 0,4421 & 1,4969 & 0,9974 & 0,0197 \\
\hline & H\&P & 0,6317 & 1,0867 & 0,9750 & 0,0612 \\
\hline \multirow{3}{*}{$65^{\circ} \mathrm{C}$} & Lewis & 0,6766 & - & 0,9651 & 0,0723 \\
\hline & Page & 0,5572 & 1,4861 & 0,9943 & 0,0292 \\
\hline & $H \& P$ & 0,7355 & 1,0787 & 0,9696 & 0,0675 \\
\hline \multirow{3}{*}{$70^{\circ} \mathrm{C}$} & Lewis & 0,7525 & - & 0,9622 & 0,0758 \\
\hline & Page & 0,6511 & 1,5339 & 0,9972 & 0,0205 \\
\hline & $H \& P$ & 0,8274 & 1,0913 & 0,9690 & 0,0685 \\
\hline
\end{tabular}
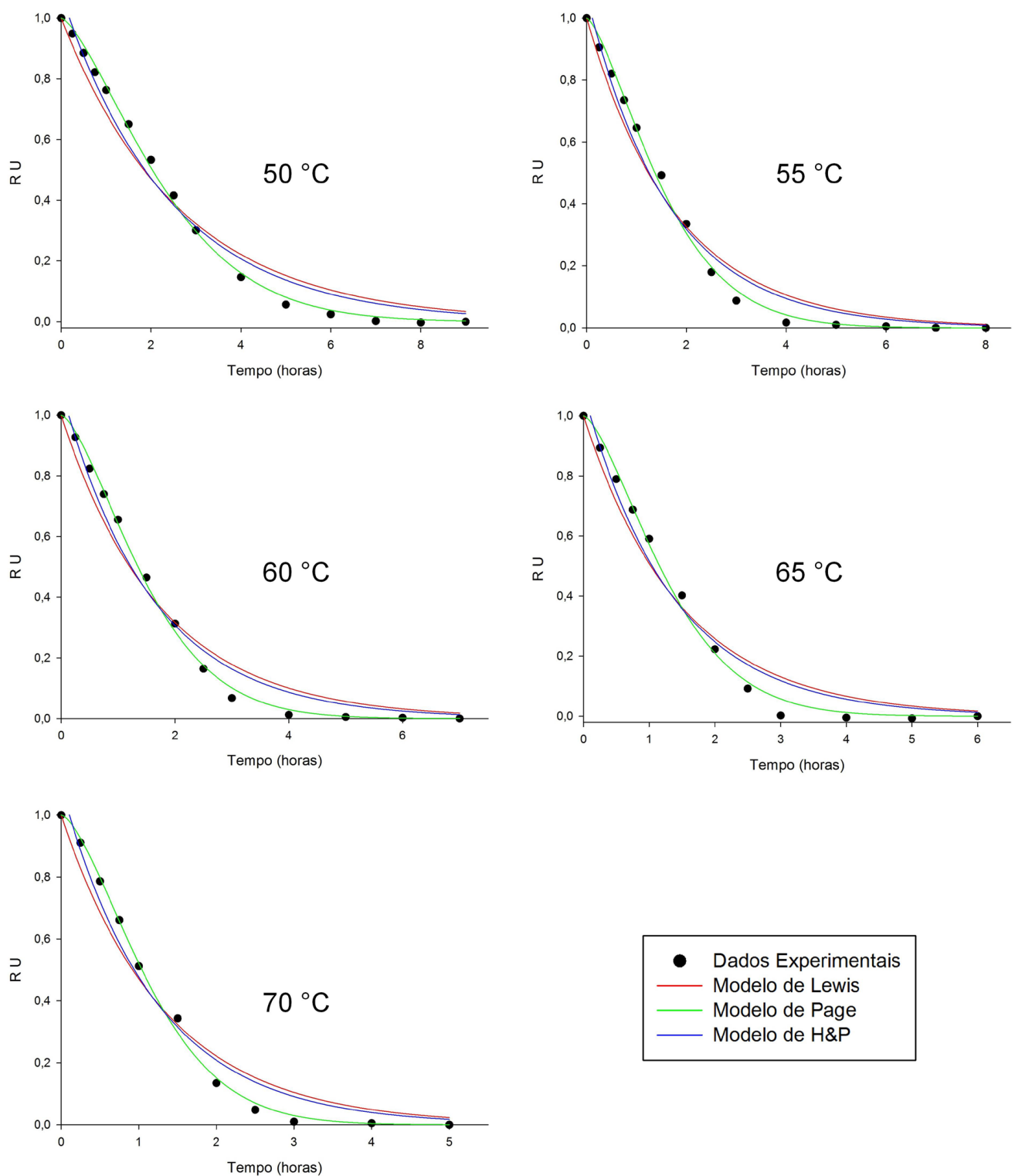

Figura 2. Curvas de secagem em leito de espuma da polpa de acerola e ajuste aos modelos de Lewis, Page e Henderson e Pabis. 
A Figura 3 apresenta as curvas de secagem em leito de espuma da polpa de acerola para as temperaturas $50^{\circ} \mathrm{C}, 55^{\circ} \mathrm{C}, 60^{\circ} \mathrm{C}, 65^{\circ} \mathrm{C}$ e $70^{\circ} \mathrm{C}$ ajustadas pelo modelo de Page. As curvas adquirem uma maior inclinação à medida que a temperatura do ar de secagem aumenta, dado o incremento de calor transferido do ar de aquecimento para o alimento.

Foi verificado que o tempo de secagem depende da temperatura, de forma que este é menor em temperaturas mais altas. Para todas as temperaturas, a razão de umidade reduziu-se rapidamente no início e, posteriormente, diminuiu lentamente à medida que se aumentou o tempo de secagem. Tais características também foram observadas na operação de secagem de nabo forrageiro (SOUSA et al., 2011) e graviola (BAPTESTINI et al., 2015).

Uma vez selecionado o modelo de Page, foi feita uma avaliação sobre a influência da temperatura sobre os parâmetros deste modelo. Para tanto, foi calculado o coeficiente de variação dos parâmetros $k$ e $n$ nas diferentes temperaturas. Para o parâmetro $k$, o coeficiente de variação foi de $32 \%$, e para o parâmetro $n$, foi de 3,2\%. Estes resultados indicam que o parâmetro $k$ variou de forma mais acentuada dentro do intervalo de temperatura e que o parâmetro $n$ teve uma pequena variação dentro do intervalo avaliado. Embora a temperatura tenha pouca influência sobre o parâmetro $n$, verificou-se uma relação linear significativa deste parâmetro com a temperatura.

Assim, para a obtenção de um modelo generalizado, em que a razão de umidade seja função da temperatura e do tempo de secagem, escolheu-se um modelo com a forma do modelo de Page, no qual os parâmetros $k$ e $n$ sejam funções da temperatura.

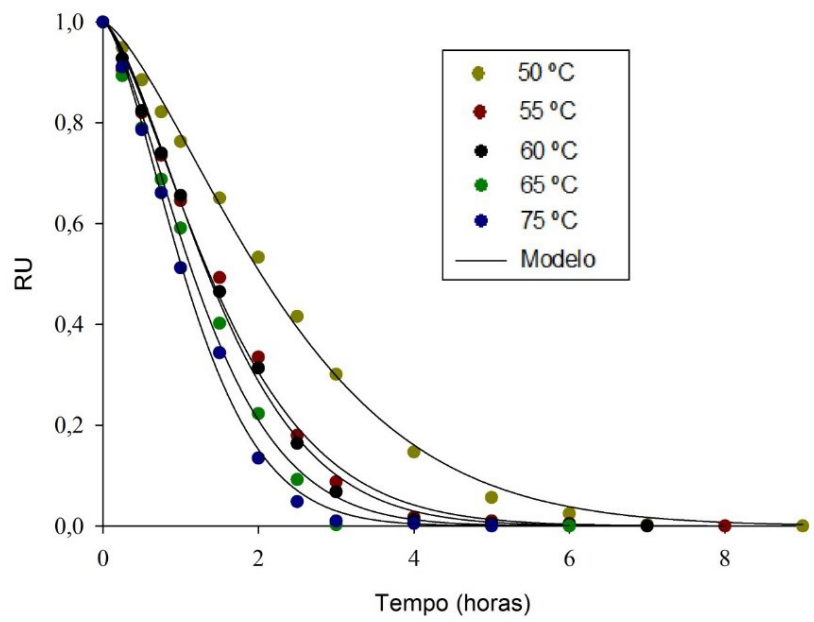

Figura 3. Curvas de secagem em leito de espuma da polpa de acerola, em diferentes temperaturas do ar de secagem ajustadas pelo modelo de Page.
Em vista de o parâmetro $k$ estar relacionado com a velocidade de secagem, este depende diretamente da difusividade efetiva do vapor d'água na secagem. A variação da difusividade efetiva em função da temperatura de secagem é comumente descrita pelo modelo de Arrhenius. Assim, foi escolhido, para representar o parâmetro k, uma função com o mesmo formato da Equação de Arrhenius. Após a substituição das equações propostas para os parâmetros ke $n$ no modelo de Page, o seguinte modelo generalizado foi obtido, conforme a Equação 5.

$R U=e^{\left[-\left(k_{1} \frac{\frac{k_{2}}{T}}{T}\right)^{(\mathrm{a} T+b)}\right]}$

em que a razão de umidade (RU) é uma função da temperatura ( $\mathrm{T})$, na escala Kelvin, e do tempo $t$, em horas; $k_{1}, k_{2}$ e $n$ são parâmetros do modelo.

Todos os valores de razão de umidade foram utilizados em todos os tempos e temperaturas de secagem, para ajuste do modelo generalizado, por meio de regressão não linear. A Tabela 3 apresenta os valores estimados para os parâmetros do modelo generalizado.

Na Figura 4, pode ser observada a dispersão dos valores de razão de umidade preditos pelo modelo generalizado versus os valores obtidos experimentalmente.

Tabela 3. Parâmetros ajustados para o modelo generalizado.

\begin{tabular}{cc} 
Parâmetro & Estimativa \\
$\mathrm{k}_{1}$ & $278345\left(\mathrm{~h}^{-1}\right)$ \\
$\mathrm{k}_{2}$ & $4439,06(\mathrm{~K})$ \\
$\mathrm{A}$ & $0,0123\left(\mathrm{~K}^{-1}\right)$ \\
$\mathrm{B}$ & $-2,6141$ \\
\hline
\end{tabular}

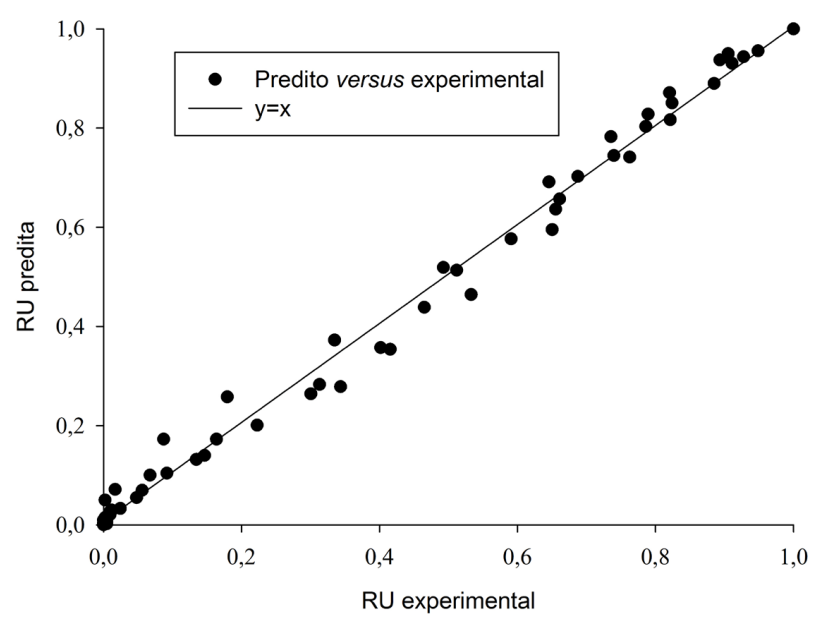

Figura 4. Valores de razão de umidade preditos pelo modelo generalizado versus os valores de razão de umidade experimentais. A linha cheia representa a função identidade. 
O coeficiente de determinação apresentado pelo modelo foi de 0,9933 , indicando um bom ajuste aos dados obtidos da secagem da polpa de acerola, em leito em espuma (MADAMBA et al., 1996). Observou-se também que os pontos se encontraram próximos e aleatoriamente distribuídos em torno da reta $\mathrm{y}=\mathrm{x}$, confirmando que $\mathrm{o}$ modelo generalizado ajustado é adequado para estimar a razão de umidade da polpa de acerola como função da temperatura do ar de secagem e do tempo de secagem, para o sistema de secagem em leito de espuma.

\subsection{Análise de umidade}

Aplicando-se a definição da razão de umidade no modelo generalizado e isolando-se a umidade, em base seca, no tempo $t$, chega-se à seguinte equação:

$\left.X_{t}=X_{e}+\left(X_{0}-X_{e}\right) e^{\left[-\left(278345 \mathrm{e}^{\frac{-4439,06}{T}}\right) t(0,0123 \mathrm{~T}-2,6141)\right.}\right]$

Nos experimentos conduzidos neste trabalho, a umidade inicial da espuma, em base úmida, apresentou um valor médio de 93,5\%, com um coeficiente de variação de $0,5 \%$. Esse valor corresponde a uma umidade em base seca $\left(X_{0}\right)$ de $14,38842 \mathrm{~kg}$ de água por quilograma de matéria seca. Verificou-se que o valor da umidade, em base seca, no equilíbrio $\left(\mathbf{X}_{\mathrm{e}}\right)$, reduziu-se exponencialmente com o aumento da temperatura. Assim, a Equação 7 foi ajustada aos dados experimentais, por meio de regressão não linear, sendo $x_{e}$ a umidade de equilíbrio em base seca (quilograma de água/ quilograma de matéria seca), e T a temperatura $(K)$.

$$
X_{e}=0,04582+3510,32 e^{-0,03389 T}
$$

A Figura 5 apresenta os valores médios de umidade de equilíbrio, em base seca, obtidos pela operação de secagem em leito de espuma, em função da temperatura do ar de secagem, e a curva simulada pelo modelo ajustado, o qual apresentou um coeficiente de determinação de 0,9541 e erro padrão da regressão de 0,003719.

Substituindo o valor médio de $\mathrm{X}_{0}$ e a equação obtida para $X_{\mathbf{e}}$ (Equação 7) na Equação 6, obtém-se a Equação 8, a qual relaciona a umidade da polpa de acerola, em base seca, em função do tempo de secagem (h) e da temperatura do ar $(\mathrm{K})$.

$X_{t}=0,04582+3510,32 e^{-0,03389 T}+$

$\left(14,3426-3510,32 \mathrm{e}^{-0,03389 \mathrm{~T}}\right) \mathrm{e}^{\left[-\left(278345 \mathrm{e}^{-\frac{-4439,06}{T}} \mathrm{ft}^{(0,0123 \mathrm{~T}-2,6141)}\right](8)\right.}$
A umidade em base úmida, $U$ (quilograma de água por $100 \mathrm{~kg}$ de matéria total, ou \%), relaciona-se com a umidade em base seca, $X$ (quilograma de água por quilograma de matéria seca), pela Equação 9.

$U_{t}=\frac{X_{t}}{1+X_{t}} \cdot 100$

Assim, a partir da utilização simultânea das Equações 8 e 9, é possível obter a umidade da espuma de acerola, em base úmida, em função do tempo de secagem (h) e da temperatura do ar (K). A Figura 6 mostra o gráfico de superfície dos valores de umidade, em base úmida, da espuma de acerola em função do tempo de secagem e da temperatura do ar, obtidos a partir das Equações 8 e 9.

A utilização de maior temperatura na operação de secagem acarretou um produto com menor valor de umidade, uma vez que, quanto maior a temperatura do ar de secagem, menor será sua umidade relativa, e, consequentemente, maior será sua capacidade de receber vapor d'água proveniente do alimento. Os resultados obtidos em todas as temperaturas, combinadas com tempos suficientes para que ocorra equilíbrio (secagem até massa constante), estão dentro do padrão de umidade estabelecido pela legislação brasileira, que preconiza que produtos de frutas secos ou desidratados apresentem valor de umidade inferior a 25\% (ANVISA, 2005).

Os produtos secos até o equilíbrio (massa constante), mesmo na menor temperatura, $50^{\circ} \mathrm{C}$, apresentaram teor de umidade suficientemente baixo, proporcionando um produto com condições aptas para o armazenamento, desde que combinado com outro método de conservação, por exemplo, embalagem metálica impermeável (SOARES et al., 2001).

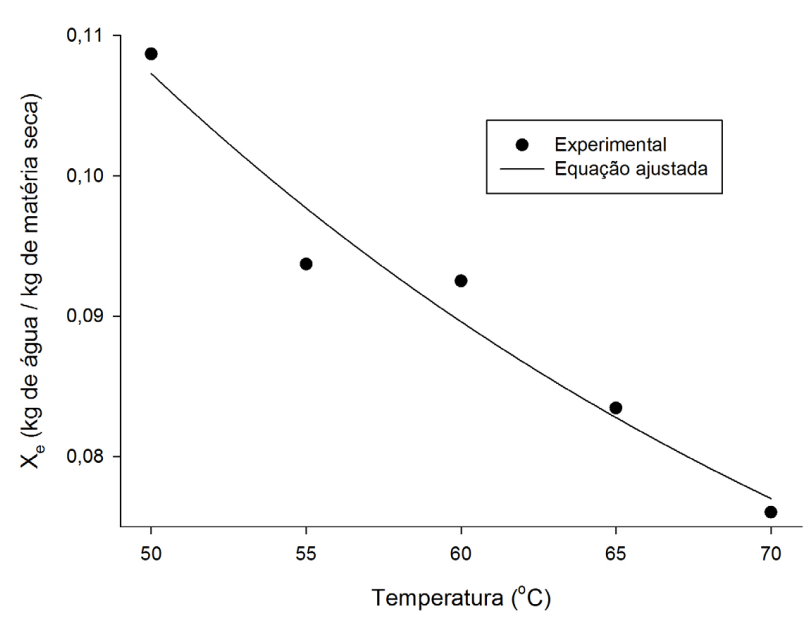

Figura 5. Variação da umidade de equilíbrio, em base seca, em função da temperatura do ar de secagem. 


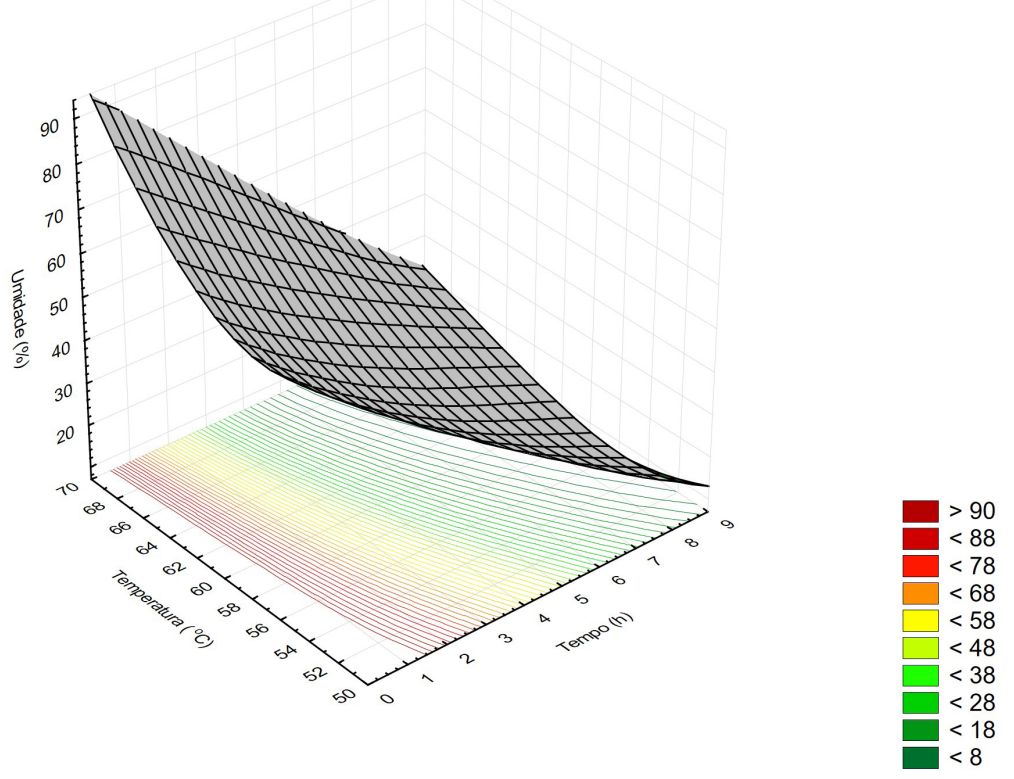

Figura 6. Umidade, em base úmida (\%), da espuma de acerola, em função do tempo (h) e da temperatura $\left({ }^{\circ} \mathrm{C}\right)$ de secagem.

\section{Conclusões}

O aumento da temperatura do ar reduziu o tempo de secagem da polpa de acerola e a umidade do produto desidratado.

O modelo de Page foi o que melhor se adequou aos dados experimentais, apresentando, para todas as temperaturas selecionadas, os maiores valores de coeficiente de determinação ajustado $(R)$, acima de 0,99, e os menores valores para o erro padrão da regressão $(S)$, abaixo de 0,03 . Os valores de $k$ e $n$ apresentaram-se entre 0,2512 e 0,6611, e 1,4221 e 1,5339, respectivamente.

O modelo generalizado ajustado apresentou alto coeficiente de determinação, indicando que este foi adequado para estimar a razão de umidade da polpa de acerola em função da temperatura do ar de secagem e do tempo de secagem.

O estudo da cinética de secagem e o ajuste de modelos matemáticos podem ser aplicados para outros alimentos e contribuir para a otimização dos processos de secagem.

\section{Referências}

AGÊNCIA NACIONAL DE VIGILÂNCIA SANITÁRIA - ANVISA. Resolução da Diretoria Colegiada - RDC n ${ }^{\circ} 272$, de 22 de setembro de 2005. Dispõe sobre o Regulamento Técnico para produtos de vegetais, produtos de frutas e cogumelos comestíveis. Diário Oficial [da] República Federativa do Brasil, Brasília, DF, 23 set. 2005.
ALVES, J. A.; VALÉRIO, E.; VILAS, D. B.; MONTEIRO, B.; BOAS, V.; SOUZA, É. C. Qualidade de produto minimamente processado à base de abóbora, cenoura, chuchu e mandioquinha-salsa. Ciência e Tecnologia de Alimentos, v. 30, n. 3, p. 625-634, 2010. http://dx. doi.org/10.1590/S0101-20612010000300009.

ASSIS, S. A.; LIMA, D. C.; OLIVEIRA, O. M. M. F. Activity of pectinmethylesterase, pectin content and vitamin $\mathrm{C}$ in acerola fruit at various stages of fruit development. Food Chemistry, v. 74, n. 2, p. 133-137, 2001. http://dx.doi.org/10.1016/S03088146(01)00104-2.

BAPTESTINI, F. M.; CORRÊA, P. C.; JUNQUEIRA, M. S.; RAMOS, A. M.; VANEGAS, J. D. B.; COSTA, C. F. Modelagem matemática da secagem de espuma de graviola. Revista Brasileira de Engenharia Agrícola e Ambiental, v. 19, n. 12, p. 293-297, 2015. http://dx.doi.org/10.1590/1807-1929/agriambi.v19n12p1203-1208.

CASARIN, F.; MENDES, C. E.; LOPES, T. J.; MOURA, N. F. Planejamento experimental do processo de secagem da amorapreta (Rubus sp.) para a produção de farinha enriquecida com compostos bioativos. Brazilian Journal of Food Technology, v. 19, p. e2016025, 2016.

CELMA, A. R.; CUADROS, F.; LÓPEZ-RODRÍGUEZ, F. Convective drying characteristics of sludge from treatment plants in tomato processing industries. Food and Bioproducts Processing, v. 90, n. 2, p. 224-234, 2011. http://dx.doi.org/10.1016/j.fbp.2011.04.003.

DANTAS, H. J.; SILVA, A. S.; LIMA, E. E.; FARIAS, P. A.; ANDRADE, M. A. Obtenção da polpa de jaca em pó pelo método de secagem em camada de espuma (foam-mat drying). In: JORNADA NACIONAL DA AGROINDÚSTRIA, 3., 2008, Bananeiras. Anais... Bananeiras: UFPB, 2008. 
Cinética de secagem de acerola em leito de espuma e ajuste de modelos matemáticos

Araújo, C. S. et al.

FELLOWS, P. J. Tecnologia do processamento de alimentos. 2. ed. Porto Alegre: Artmed, 2006. 602 p.

HENDERSON, S. M.; PABIS, S. Grain drying theory I: temperature effect on drying coefficient. Journal of Agricultural Research Engineering, v. 12, p. 732-736, 1962

HOFSKY, A. V.; GOMES, J. P.; BARROS NETO, A. L.; SILVA, F. L. H.; ALMEIDA, F. A. C. Cinética de secagem de abacaxi cv pérola em fatias. Revista Brasileira de Produtos Agroindustriais, v. 11, n. 2, p. 123-128, 2009. http://dx.doi.org/10.15871/1517-8595/ rbpa.v11n2p123-128

KADAM, D. M.; BALASUBRAMANIAN, S. Foam mat drying of tomato juice. Journal of Food Processing and Preservation, v. 35, n. 4, p. 488-495, 2011. http://dx.doi.org/10.1111/j.17454549.2010.00492.x.

KALETA, A.; GÓRNICKI, K.; WINICZENKO, R.; CHOJNACKA, A. Evaluation of drying models of apple (var. Ligol) dried in a fluidized bed dryer. Energy Conversion and Management, v. 67, p. 179-185, 2013. http://dx.doi.org/10.1016/j.enconman.2012.11.011.

KANDASAMY, P.; VARADHARAJU, N.; KALEMULLAH, S.; RANABIR, $M$. Production of papaya powder under foam-mat drying using methyl cellulose as foaming agent. Asian Journal of Food and Agro-Industry, v. 5, n. 5, p. 374-387, 2012.

KARIM, A. A.; WAI, C. C. Foam-mat drying of starfruit (Averrhoa carambola L.) puree. Stability and air drying characteristics. Food Chemistry, v. 64, n. 3, p. 337-343, 1999. http://dx.doi. org/10.1016/S0308-8146(98)00119-8.

LEWIS, W. K. The rate of drying of solid materials. Journal of Industrial and Engineering Chemistry, v. 13, n. 5, p. 427-433, 1921. http://dx.doi.org/10.1021/ie50137a021.

MADAMBA, P. S.; DRISCOLL, R. H.; BUCKLE, K. E. The thin layer drying characteristic of garlic slices. Journal of Food Engineering, v. 29, n. 1, p. 75-97, 1996. http://dx.doi. org/10.1016/0260-8774(95)00062-3.

MEZADRI, T.; VILLAÑO, D.; FERNÁNDEZ-PACHÓN, M. S.; GARCÍAPARRILLA, M. C.; TRONCOSO, A. M. Antioxidant compounds and antioxidant activity in acerola (Malpighia emarginata DC.) fruits and derivatives. Journal of Food Composition and
Analysis, v. 21, n. 4, p. 282-290, 2008. http://dx.doi.org/10.1016/j. jfca.2008.02.002.

MEZIANE, S. Drying kinetics of olive pomace in a fluidized bed dryer. Energy Conversion and Management, v. 52, n. 3, p. 16441649, 2011. http://dx.doi.org/10.1016/j.enconman.2010.10.027.

MOREIRA, T. B.; ROCHA, É. M. F. F.; AFONSO, M. R. A.; COSTA, J. M. C. Comportamento das isotermas de adsorção do pó da polpa de manga liofilizada. Revista Brasileira de Engenharia Agrícola e Ambiental, v. 17, n. 10, p. 1093-1098, 2013. http:// dx.doi.org/10.1590/S1415-43662013001000011.

PAGE, G. E. Factors influencing the maximum rates of air drying shelled corn in thin layers. 1949. Dissertação (Mestrado)Purdue University, West Lafayette, 1949.

PINTO, E. G. Caracterização da espuma de jenipapo (Genipa americana L.) com diferentes aditivos visando à secagem em leito de espuma. 2009. 69 f. Dissertação (Mestrado em Engenharia de Alimentos)-Universidade Estadual do Sudoeste da Bahia, Itapetinga, 2009.

RAJKUMAR, P.; KAILAPPAN, R.; VISWANATHAN, R.; RAGHAVAN, G. S. V.; RATTI, C. Foam mat drying of Alphonso mango pulp. Drying Technology, v. 25, n. 2, p. 357-365, 2007. http://dx. doi. org/10.1080/07373930601120126.

SOARES, E. C. Caracterização de aditivos para secagem de araça-boi (Eugenia stipitata Mc Vaugh) em leito de espuma. 2009. 89 f. Dissertação (Mestrado em Engenharia de Alimentos)Universidade Federal da Bahia, Itapetininga, 2009.

SOARES, E. C.; OLIVEIRA, G. S. F.; MAIA, G. A.; MONTEIRO, J. C. S.; SILVA JUNIOR, A.; FILHO, M. S. Desidratação da polpa de acerola (Malpighia emarginata D.C.) pelo processo foam-mat. Ciência e Tecnologia de Alimentos, v. 21, n. 2, p. 164-170, 2001. http://dx.doi.org/10.1590/S0101-20612001000200008.

SOUSA, K. A.; RESENDE, O.; CHAVES, T. H.; COSTA, L. M. The drying kinetics of forage turnips (Raphanus sativus L. ). Revista Ciência Agronômica, v. 42, n. 4, p. 883-892, 2011. http://dx. doi. org/10.1590/S1806-66902011000400009.

VIEIRA, A. H.; FIGUEIRÊDO, R. M. F.; QUEIROZ, A. J. M. Isotermas de adsorção de umidade da pitanga em pó. Revista de Biologia e Ciências da Terra, v. 7, p. 11-20, 2007. 